\title{
344 - Study protocol for a feasibility RCT of Club Connect, a healthy brain ageing cognitive training program for older adults with depression
}

Claudia Woolf; Leanne Kaplan; Sharon L Naismith, BA, Hons, DPsych, MA; David Burke; Louisa Margaret Norrie, Psychiatrist; Loren Mowszowski

Background: Current treatments for Major Depression are only moderately effective. In fact, $20-30 \%$ do not achieve full recovery despite multiple interventions. Interestingly, while it is well recognised that cognitive impairment is associated with Major Depression, available treatments do not address cognitive impairment. In this regard, cognitive training (CT) represents a promising intervention, however CT is not typically offered in public health settings.

Aim: To evaluate the feasibility of a blinded, randomised controlled trial of group-based CT in a hospital sample of older adults with clinically significant depressive symptoms or history of a major depressive episode within the last five years, and in doing so, to adapt and translate research findings to the clinical setting.

Methods: 40 older adults, aged at least 65 years, with depression or a history of depression, and without dementia were randomly allocated to Club Connect, a 10-week group-based healthy brain ageing CT program, or a waitlist, treatment-as-usual control group. Baseline assessment including review by a psychogeriatrician and a brief neuropsychological assessment was completed within a fortnight of the intervention starting, and follow-up assessment was completed within a fortnight of the intervention ceasing. Primary outcomes included feasibility of trial design, tolerability of the intervention, and acceptability of random allocation and data collection procedures (as perceived by both participants and clinicians). We also examined the most sensitive clinical outcomes and measurement tools to inform larger scale trials.

Significance: The current health, social and economic costs of late-life depression, especially in those with concomitant cognitive impairment, renders the holistic treatment of depression in older adults a public health priority. CT represents an efficacious therapeutic intervention in this regard, however, there appears to be a paucity of CT programs being offered in public health settings. This trial represents the first step in addressing the 'implementation gap' that exists between care that is known to be effective and care that is delivered. We must recognise the need to evaluate not only health outcomes, but also to perform formative evaluations that assess the extent to which implementation is effective in order to optimise intervention benefits, to prolong sustainability, and to promote dissemination of findings. 